\title{
Justification of the Fast Multipole Method for the Stokes System. Part II: Exterior Domain Problems
}

\author{
T. S. Samrowski*
}

\begin{abstract}
This is the second part of the paper, which justifies the solution of the two-dimensional Stokes equations using the fast multipole method (FMM) of Greengard and Rokhlin. In addition to the interior Dirichlet problem, which was considered in the previous part, we are concerned with exterior domain problems of Dirichlet and Neumann kinds. For the solution of the boundary value problems, we choose a potential ansatz and show that for the reduction of the computational costs, FMM can be used. Therefor, we find a complex representation of the solution and provide the statements about the corresponding multipole and Taylor expansions, as well as the appropriate translation, rotation and conversion operators. Numerical experiments illustrate the performance of FMM for the proposed cases.
\end{abstract}

Key words and phrases: Stokes system, fast multipole method, hydrodynamical potentials

\section{Introduction}

For the solution of the stationary linearized Navier-Stokes system (so-called stationary Stokes equations' system), different integral equation formulations have been already proposed. In $[12,16]$, e.g., an extensive theory based on complex variables has been developed. Khoromskij et al. presented in [13] a technique of almost linear complexity for solving elliptical partial differential equations based on their reduction to the interface. In the case of the Stokes equations, this interface reduction method is based on either the stream function-vorticity formulation or on the use of the special Poincaré-Steklov operator. An alternative approach, using fundamental solution and hydrodynamical potentials, can be found in [1, 14, 21] and provides the analytical foundation for our discussion.

We restrict our attention to the classical boundary value problems of the Stokes equations in exterior domains. By the methods presented in [1, 29], we obtain a representation of the solution in form of hydrodynamical potentials, whose unknown densities are solutions of uniquely solvable boundary integral equations. For the numerical solution of these integral equations with $m$ points in the discretization of the boundary, a dense, non-symmetrical linear system have to be solved. Direct inversion of such a system requires $\mathcal{O}\left(\mathrm{m}^{3}\right)$ operations. The most work intensive part by the application of the iterative methods is the computation of matrix-vector products. Direct calculation of a matrix-vector product requires $\mathcal{O}\left(\mathrm{m}^{2}\right)$ operations. Since one uses the fast multipole method $[2,5,23]$ to compute matrix-vector products, the computational costs can be reduced up to $\mathcal{O}\left(m \log ^{\alpha} m\right)$. Furthermore, an iterative method with FMM does not require storage of a dense matrix $[15,22,23]$.

\footnotetext{
*Corresponding author: e-mail: tatiana.samrowski@math.uzh.ch, Phone: $\quad+41446355854$, Fax: $+44446355705$
} 
In this paper, we present a new representation of hydrodynamical tensors based on complex variables, verify the corresponding multipole and Taylor expansions and provide the theorems about the translation and conversion of these expansions. All these analytical preliminaries allow us to use the fast multipole method for the numerical solution of our boundary integral equations and for the computation of the approximate hydrodynamical potentials. For $m$ nodes in the discretization of the boundary, the velocity part of hydrodynamical potentials consists of $m^{2}$ symmetrical blocks of the size $2 \times 2$ with the elements having three different prescription. Therefore, we will need several FMM cycles to calculate one matrix-vector product in our case. As it was in the case of the interior Dirichlet problem presented in [27], this method essentially reduces the computational costs, which will be demonstrated by numerical test computations.

In the next section, we briefly review the analytical foundation concerning the solvability of the boundary value problem of the Stokes equations in exterior domains and present the corresponding integral equation formulations. In Section 3, we deduce representations of hydrodynamical single layer potential tensor and of its normal stress based on complex variables. Section 4 contains a detail description of the analytical preliminaries, which are necessary for the application of the fast multipole method. The performance of the proposed method is illustrated with numerical examples in Section 5.

\section{Solvability of the exterior boundary value problems of the Neumann and Dirichlet kind for the system of Stokes equa- tions}

We consider the two-dimensional Stokes system:

$$
-\Delta \mathbf{u}+\nabla p=\mathbf{0} \quad \text { in } \quad G^{*}, \quad \nabla \cdot \mathbf{u}=0 \quad \text { in } \quad G^{*}
$$

with boundary conditions of two types:

$$
\begin{array}{lrll}
\text { Neumann: } & T \mathbf{n}=\mathbf{b} & \text { on } & \partial G^{*} \\
\text { Dirichlet: } & \mathbf{u}=\mathbf{b} & \text { on } & \partial G^{*}
\end{array}
$$

The domain $G^{*} \subset \mathbb{R}^{2}$ with boundary $\partial G^{*} \in \mathbb{C}^{2}$, in which system (1) is to be solved, will be a domain exterior to a simple closed curve. Here $\mathbf{u}$ is the velocity vector, $p$ the (scalar) pressure of a viscous incompressible fluid with conservative external forces, $\mathbf{n}$ denotes the outward unit normal vector on $\partial G^{*}$, and $T$ is the stress tensor defined by

$$
T_{i j}:=-p \delta_{i j}+\left(\frac{\partial u_{i}}{\partial x_{j}}+\frac{\partial u_{j}}{\partial x_{i}}\right), \quad i, j=1,2
$$

with $\delta_{i j}$ being Kronecker delta. Physically, the Neumann condition corresponds to the prescribing force distribution on the boundary. $\mathbf{u}$ and $\mathbf{b}$ are two-dimensional vector functions in $G^{*}$ and on $\partial G^{*}$, respectively, $\Delta$ denotes the Laplacian, $\nabla$ the gradient and $\nabla \cdot$ the divergence in $\mathbb{R}^{2}$. We assume that the boundary value $\mathbf{b}$ is given continuously on $\partial G^{*}$.

In the following two lemmata, we outline the main results for the solvability of the Stokes system in cases (2) and (3) without detailed investigation of the corresponding problems (one can find it, e.g., in [14], [21] and [29]). 
Lemma 2.1 For any continuous vector function $\mathbf{b}$ with the property

$$
\int_{\partial G^{*}} \mathbf{b} \text { do }=0
$$

the exterior Neumann problem for the Stokes equations

$$
-\Delta \mathbf{u}+\nabla p=\mathbf{0} \quad \text { in } G^{*}, \quad \nabla \cdot \mathbf{u}=0 \quad \text { in } G^{*}, \quad T \mathbf{n}=\mathbf{b} \quad \text { on } \partial G^{*}
$$

has a solution $(\mathbf{u}, p)$ which satisfies the decay conditions

$$
\begin{array}{rlll}
\mathbf{u}(\mathbf{x}) & =\mathcal{O}(1) & \text { as } & |\mathbf{x}| \longrightarrow \infty \\
\nabla \mathbf{u}(\mathbf{x}) & =\mathcal{O}\left(|\mathbf{x}|^{-2}\right) & \text { as } & |\mathbf{x}| \longrightarrow \infty \\
p(\mathbf{x}) & =\mathcal{O}\left(|\mathbf{x}|^{-2}\right) & \text { as } & |\mathbf{x}| \longrightarrow \infty
\end{array}
$$

This solution is unique to within a constant vector $\mathbf{c}$, and if $\mathbf{u} \longrightarrow 0$ for $|\mathbf{x}| \longrightarrow \infty$, then the solution of the exterior Neumann problem for the Stokes equations is unique.

If we seek a solution of the problem (6) as in [21] in the form

$$
\mathbf{u}=\nabla \ln |\mathbf{x}| \int_{\partial G} \mathbf{q}(\mathbf{y}) \mathbf{n}(\mathbf{y}) \text { do }+E \mathbf{q}(\mathbf{x}), \quad p=\frac{1}{2 \pi} \int_{\partial G} \frac{\mathbf{x}-\mathbf{y}}{|\mathbf{x}-\mathbf{y}|^{2}} \mathbf{q}(\mathbf{y}) \text { do, } \quad \mathbf{x} \in \mathbf{G}^{*},
$$

where

$$
E \mathbf{q}(\mathbf{x}):=\int_{\partial G} \widetilde{E}(\mathbf{x}-\mathbf{y}) \mathbf{q}(\mathbf{y}) \mathrm{ds}, \quad \mathbf{x} \in \mathbf{G}^{*}
$$

is the velocity part of the hydrodynamical single layer potential with the corresponding $2 \times$ $2-$ matrix

$$
\widetilde{E}_{i j}(\mathbf{x}-\mathbf{y})=\frac{1}{4 \pi} \frac{\left(x_{i}-y_{i}\right)\left(x_{j}-y_{j}\right)}{|\mathbf{x}-\mathbf{y}|^{2}}-\delta_{i j} \ln |\mathbf{x}-\mathbf{y}|, \quad i, j=1,2,
$$

then the unknown density $\mathbf{q}$ can be obtained using well known continuity and jump relations of the hydrodynamical potentials (see, e.g., [14]) from the following uniquely solvable (cf. [21]) system of boundary integral equations:

$$
-\frac{1}{2} \mathbf{q}(\mathbf{x})-H \mathbf{q}(\mathbf{x})+\left(\int_{\partial \mathbf{G}} \mathbf{q}(\mathbf{y}) \cdot \mathbf{n}(\mathbf{y}) \mathrm{do}_{\mathbf{y}}\right) \mathbf{g}(\mathbf{x})=\mathbf{b}(\mathbf{x}), \quad \mathbf{x} \in \partial G^{*},
$$

where

$$
H \mathbf{q}(\mathbf{x}):=\int_{\partial G} \widetilde{H}(\mathbf{x}, \mathbf{y}) \mathbf{q}(\mathbf{y}) \mathrm{do}_{\mathbf{y}}
$$

with

$$
\widetilde{H}_{i j}(\mathbf{x}, \mathbf{y})=T_{i j}(\widetilde{E}(\mathbf{x}-\mathbf{y})) \mathbf{n}(\mathbf{x})=\frac{1}{\pi} \frac{\left(x_{i}-y_{i}\right)\left(x_{j}-y_{j}\right)(\mathbf{x}-\mathbf{y}) \cdot \mathbf{n}(\mathbf{x})}{|\mathbf{x}-\mathbf{y}|^{4}}, \quad i, j=1,2,
$$

and

$$
g_{i}(\mathbf{x}):=\left.\left\{T_{i j}(\nabla \ln |\mathbf{x}|) n_{j}\right\}\right|_{\partial G}=2 n_{j} \frac{\partial^{2}}{\partial x_{i} \partial x_{j}} \ln |\mathbf{x}|, \quad i, j=1,2 .
$$

For the numerical solution of (11), one can parameterize these equations with $\gamma:[0,1] \longrightarrow \partial G$ and discretize them using, e.g., the Nyström method, by $m$ discretization points. Setting 
$h:=\frac{1}{m}$, one obtains for (11) the following system of linear equations, which can be solved, e.g., by an iterative method:

$$
\begin{aligned}
-\frac{1}{2} \widetilde{\mathbf{q}}(i h) & -h \sum_{i \neq j=1}^{m} \widetilde{H_{\gamma}}(i h, j h) \widetilde{\mathbf{q}}(j h)-h \mathcal{K} \widetilde{\mathbf{q}}(j h) \\
& +h \widetilde{\mathbf{g}}(i h) \sum_{j=1}^{m} \widetilde{\mathbf{f}}(j h) \cdot \widetilde{\mathbf{n}_{\gamma}}(j h)=\widetilde{\mathbf{b}}(i h), \quad i, j=1, \ldots, m
\end{aligned}
$$

with

$$
\begin{gathered}
\widetilde{\mathbf{q}}(i h):=\mathbf{q}(\gamma(i h)), \widetilde{\mathbf{b}}(i h):=\mathbf{b}(\gamma(i h)), \widetilde{\mathbf{g}}(i h):=\mathbf{g}(\gamma(i h)), \quad \widetilde{\mathbf{n}_{\gamma}}(j h):=\mathbf{n}(\gamma(j h))|\dot{\gamma}(j h)|, \\
\widetilde{H_{\gamma}}(i h, j h):=\widetilde{H}(i h, j h)|\dot{\gamma}(j h)|,
\end{gathered}
$$

and a curvature matrix $\mathcal{K}$, which can be explicit specified for a smooth boundary.

Lemma 2.2 The exterior Dirichlet problem of the Stokes equations

$$
-\Delta \mathbf{u}+\nabla p=\mathbf{0} \quad \text { in } G^{*}, \quad \nabla \cdot \mathbf{u}=0 \quad \text { in } \quad G^{*}, \quad \mathbf{u}=\mathbf{b} \quad \text { on } \quad \partial G^{*}
$$

has a unique solution (u, $p$ ) in the class of functions, which have the property (7).

As in [29], we choose for the solution of (16) the potential ansatz and obtain for the velocity part of the solution the representation

$$
\mathbf{u}(\mathbf{x}):=D \mathbf{q}(\mathbf{x})-\eta E M \mathbf{q}(\mathbf{x})-\alpha \int_{\partial G^{*}} \mathbf{q d o}, \quad 0<\eta \in \mathbb{R}, \quad 0 \neq \alpha \in \mathbb{R} .
$$

Here

$$
D \mathbf{q}(\mathbf{x}):=\int_{\partial G} \widetilde{D}(\mathbf{x}, \mathbf{y}) \mathbf{q}(\mathbf{y}) \operatorname{do}_{\mathbf{y}}
$$

denotes the velocity part of the hydrodynamical double layer potential with the corresponding $2 \times 2$-matrix

$$
\widetilde{D}_{i j}(\mathbf{x}, \mathbf{y}):=-\frac{1}{\pi} \frac{\left(x_{i}-y_{i}\right)\left(x_{j}-y_{j}\right)(\mathbf{x}-\mathbf{y}) \cdot \mathbf{n}(\mathbf{y})}{|\mathbf{x}-\mathbf{y}|^{4}}, \quad i, j=1,2,
$$

$E M \mathbf{q}(\mathbf{x})$ is velocity part of the hydrodynamical single layer potential, which is applied to the image of the projector $M: C\left(\partial G^{*}\right)^{2} \longrightarrow C\left(\partial G^{*}\right)^{2}$ defined by

$$
\mathbf{q} \longrightarrow M \mathbf{q}:=\mathbf{q}-\mathbf{q}_{M}
$$

with the surface mean value

$$
\mathbf{q}_{M}:=\frac{1}{\left|\partial G^{*}\right|} \int_{\partial G^{*}} \mathbf{q} \text { do, } \quad\left|\partial G^{*}\right|=\int_{\partial G^{*}} 1 \text { do. }
$$

The unknown density $\mathbf{q}$ from (17) can be found as a unique solution of the following system of boundary integral equations (cf. [29]):

$$
-\frac{1}{2} \mathbf{q}(\mathbf{x})+D \mathbf{q}(\mathbf{x})-\eta E M \mathbf{q}(\mathbf{x})-\alpha\left|\partial G^{*}\right|\left(\mathbf{I}_{2}-M\right) \mathbf{q}(\mathbf{x})=\mathbf{b}(\mathbf{x}), \quad \mathbf{x} \in \partial G^{*}
$$


with $0<\eta \in \mathbb{R}, 0 \neq \alpha \in \mathbb{R}$ and $\mathbf{I}_{\mathbf{2}}$ is the $2 \times 2$-identity matrix.

The most costly part by the iterative solution of systems (11) and (20), as well as by the approximative computation of the hydrodynamical potentials in (8) and (17), is the calculation of matrix-vector products. This procedure requires $\mathcal{O}\left(\mathrm{m}^{2}\right)$ multiplications, but the computational costs can be essentially reduced if the corresponding matrix components $\mathbf{k}\left(\mathbf{x}^{i}, \mathbf{x}^{j}\right)$ can be approximated by the term with separable variables:

$$
\mathbf{k}\left(\mathbf{x}^{i}, \mathbf{x}^{j}\right) \approx \sum_{l=1}^{M} \mathbf{u}_{l}\left(\mathbf{x}^{i}\right) \mathbf{v}_{l}\left(\mathbf{x}^{j}\right)
$$

In this case, the left hand side of (14) is of the form

$$
\left(\sum_{j=1}^{m} \mathbf{k}\left(\mathbf{x}^{i}, \mathbf{x}^{j}\right) \mathbf{f}\left(\mathbf{x}^{j}\right)\right)_{i} \approx\left(\sum_{l=1}^{M} \mathbf{u}_{l}\left(\mathbf{x}^{i}\right) \sum_{j=1}^{m} \mathbf{v}_{l}\left(\mathbf{x}^{j}\right) \mathbf{f}\left(\mathbf{x}^{j}\right)\right)_{i} .
$$

Product (22) only requires $\mathcal{O}(M m)$ floating operations, which is much faster then $\mathcal{O}\left(m^{2}\right)$ by increasing $m$. This idea is used by the fast multipole method (see, e.g., [7], [23]). Hence, if we find for the matrices (10), (13) and (19) the representation of the form (21), then we will be able to use FMM by the calculation of hydrodynamical potentials and assentially reduce the computational costs by the numerical solution of our exterior problems (6). We have already done it in the case of the interior Dirichlet problem for the Stokes equations in [27], where we presented a suitable representation of the velocity part of the hydrodynamical double layer tensor (19) and derived the corresponding multipole and Taylor expansion, as well as its shifting

and converting operators. Therefore, we will only discuss in the following the hydrodynamical single layer tensor (10) and its normal stress tensor (13).

\section{A complex valued representation of the hydrodynamical po- tentials}

Let $z, z_{0}, z_{1}, \ldots, z_{m} \in \mathbb{C}$ be given points in the complex plane. We will not make any distinction between a point $\mathbf{x}=\left(x_{1}, x_{2}\right) \in \mathbb{R}^{2}$ and a point $x_{1}+i x_{2}=z \in \mathbb{C}$, and set for $\mathbf{x}, \mathbf{y}, \mathbf{n} \in \mathbb{R}^{2}$

$$
\begin{aligned}
\mathbf{x}=\left(x_{1}, x_{2}\right) & \leftrightarrow \quad z=x_{1}+i x_{2}, \\
\mathbf{y}=\left(y_{1}, y_{2}\right) & \leftrightarrow \quad z_{0}=y_{1}+i y_{2}, \\
\mathbf{n}(\mathbf{x})=\left(n_{1}(\mathbf{x}), n_{2}(\mathbf{x})\right) & \leftrightarrow \quad N=n_{1}(\mathbf{x})+i n_{2}(\mathbf{x}), \text { and } M:=n_{2}(\mathbf{x})+i n_{1}(\mathbf{x}) .
\end{aligned}
$$

We write $\bar{N}$ and $\bar{M}$ for the conjugate of $N$ and $M$, and denote by $\mathcal{R} e z$ and $\mathcal{I} m z$ the real and the imaginary part of any $z \in \mathbb{C}$, respectively. With this notation, we find:

$$
\begin{aligned}
& \mathcal{R} e\left(z-z_{0}\right) \mathcal{R} e \frac{1}{z-z_{0}}=\left(x_{1}-y_{1}\right) \mathcal{R} e \frac{\left(x_{1}-y_{1}\right)-i\left(x_{2}-y_{2}\right)}{\left|z-z_{0}\right|^{2}}=\frac{\left(x_{1}-y_{1}\right)^{2}}{|\mathbf{x}-\mathbf{y}|^{2}} \\
& \mathcal{I} m\left(z-z_{0}\right) \mathcal{R} e \frac{1}{z-z_{0}}=\frac{\left(x_{1}-y_{1}\right)\left(x_{2}-y_{2}\right)}{|\mathbf{x}-\mathbf{y}|^{2}}, \\
& \mathcal{I} m\left(z_{0}-z\right) \mathcal{I} m \frac{1}{z-z_{0}}=\frac{\left(x_{2}-y_{2}\right)^{2}}{|\mathbf{x}-\mathbf{y}|^{2}}, \\
& \mathcal{R} e\left(\log \left(z-z_{0}\right)\right)=\mathcal{R} e\left(\ln \left|z-z_{0}\right|+i\left(\arg \left(z-z_{0}\right)+2 k \pi\right)\right)=\ln |\mathbf{x}-\mathbf{y}| .
\end{aligned}
$$


Then, we obtain for the velocity part of the hydrodynamic single layer tensor the following complex representation:

$$
\begin{aligned}
& E_{11}(\mathbf{x}-\mathbf{y}) \quad \leftrightarrow \quad E_{11}\left(z-z_{0}\right)=\frac{1}{4 \pi}\left(\mathcal{R} e\left(z-z_{0}\right) \mathcal{R} e \frac{1}{z-z_{0}}-\mathcal{R} e\left(\log \left(z-z_{0}\right)\right)\right) \\
& E_{12}(\mathbf{x}-\mathbf{y}) \quad \leftrightarrow \quad E_{12}\left(z-z_{0}\right)=E_{21}\left(z-z_{0}\right)=\frac{1}{4 \pi} \mathcal{I} m\left(z-z_{0}\right) \mathcal{R} e \frac{1}{z-z_{0}}, \\
& E_{22}(\mathbf{x}-\mathbf{y}) \quad \leftrightarrow \quad E_{22}\left(z-z_{0}\right)=\frac{1}{4 \pi}\left(\mathcal{I} m\left(z_{0}-z\right) \mathcal{I} m \frac{1}{z-z_{0}}-\mathcal{R} e\left(\log \left(z-z_{0}\right)\right)\right) .
\end{aligned}
$$

In order to find a complex version of the normal stress tensor from (13), which is corresponded to the hydrodynamical single layer potential, we note that

$$
\begin{gathered}
\mathcal{R} e \frac{N}{z-z_{0}}=\frac{\left|z-z_{0}\right|^{2}}{\left|z-z_{0}\right|^{2}} \mathcal{R} e \frac{\left(n_{1}+i n_{2}\right)\left(\left(x_{1}-y_{1}\right)-i\left(x_{2}-y_{2}\right)\right)}{\left|z-z_{0}\right|^{2}} \\
=\frac{n_{1}\left(x_{1}-y_{1}\right)^{3}+n_{1}\left(x_{1}-y_{1}\right)\left(x_{2}-y_{2}\right)^{2}}{\left|z-z_{0}\right|^{4}}+\frac{n_{2}\left(x_{1}-y_{1}\right)^{2}\left(x_{2}-y_{2}\right)+n_{2}\left(x_{2}-y_{2}\right)^{3}}{\left|z-z_{0}\right|^{4}}, \\
\begin{aligned}
\mathcal{R} e \frac{N}{\left(z-z_{0}\right)^{2}} & =\mathcal{R} e \frac{\left(n_{1}+i n_{2}\right)\left(\left(x_{1}-y_{1}\right)-i\left(x_{2}-y_{2}\right)\right)^{2}}{\left|z-z_{0}\right|^{4}} \\
& =\frac{n_{1}\left(x_{1}-y_{1}\right)^{2}-n_{1}\left(x_{2}-y_{2}\right)^{2}+2 n_{2}\left(x_{1}-y_{1}\right)\left(x_{2}-y_{2}\right)}{\left|z-z_{0}\right|^{4}},
\end{aligned}
\end{gathered}
$$

and

$$
\mathcal{R} e \frac{\bar{N}}{\left(z-z_{0}\right)^{2}}=\frac{n_{1}\left(x_{1}-y_{1}\right)^{2}-n_{1}\left(x_{2}-y_{2}\right)^{2}-2 n_{2}\left(x_{1}-y_{1}\right)\left(x_{2}-y_{2}\right)}{\left|z-z_{0}\right|^{4}} .
$$

As well as

$$
\frac{1}{4} \mathcal{R} e\left(z-z_{0}\right)\left(\mathcal{R} e \frac{N}{\left(z-z_{0}\right)^{2}}-\mathcal{R} e \frac{\bar{N}}{\left(z-z_{0}\right)^{2}}\right)=\frac{n_{2}\left(x_{1}-y_{1}\right)^{2}\left(x_{2}-y_{2}\right)}{\left|z-z_{0}\right|^{4}},
$$

and

$$
\frac{1}{4} \mathcal{I} m\left(z-z_{0}\right)\left(\mathcal{R} e \frac{M}{\left(z-z_{0}\right)^{2}}-\mathcal{R} e \frac{\bar{M}}{\left(z-z_{0}\right)^{2}}\right)=\frac{n_{1}\left(x_{1}-y_{1}\right)\left(x_{2}-y_{2}\right)^{2}}{\left|z-z_{0}\right|^{4}} .
$$

With (25)-(29), we obtain the following complex representation of (13):

$$
\begin{aligned}
& \widetilde{H}_{11}(\mathbf{x}, \mathbf{y}) \leftrightarrow \widetilde{H}_{11}\left(z-z_{0}\right)=\frac{1}{4 \pi}\left(\mathcal{R} e \frac{4 N}{z-z_{0}}-\mathcal{R} e\left(z-z_{0}\right) \mathcal{R} e \frac{N-\bar{N}}{\left(z-z_{0}\right)^{2}}+\mathcal{I} m\left(z-z_{0}\right) \mathcal{R} e \frac{3 \bar{M}+M}{\left(z-z_{0}\right)^{2}}\right), \\
& \widetilde{H}_{12}(\mathbf{x}, \mathbf{y}) \leftrightarrow \widetilde{H}_{12}\left(z-z_{0}\right)=\widetilde{H}_{21}\left(z-z_{0}\right)=\frac{1}{4 \pi}\left(\mathcal{R} e\left(z-z_{0}\right) \mathcal{R} e \frac{M-\bar{M}}{\left(z-z_{0}\right)^{2}}+\mathcal{I} m\left(z-z_{0}\right) \mathcal{R} e \frac{N-\bar{N}}{\left(z-z_{0}\right)^{2}}\right), \\
& \widetilde{H}_{22}(\mathbf{x}, \mathbf{y}) \leftrightarrow \widetilde{H}_{22}\left(z-z_{0}\right)=\frac{1}{4 \pi}\left(\mathcal{R} e \frac{4 N}{z-z_{0}}-\mathcal{R} e\left(z-z_{0}\right) \mathcal{R} e \frac{3 N+\bar{N}}{\left(z-z_{0}\right)^{2}}-\mathcal{I} m\left(z-z_{0}\right) \mathcal{R} e \frac{M-\bar{M}}{\left(z-z_{0}\right)^{2}}\right) .
\end{aligned}
$$

A complex representation of the double layer tensor (19) is similar to the representation (30) of the normal stress tensor and has already been derived and discussed in [27]. 


\section{Analytical investigation of the hydrodynamical tensors}

The iterative solution of the linear system (14) requires the evaluation of the finite sums of the types

$$
\mathcal{R} e\left(N \sum_{j=1}^{m} \frac{q_{j}}{z-z_{j}}\right) \quad \text { and } \quad \sum_{j=1}^{m} q_{j} \mathcal{R} e\left(z-z_{j}\right) \mathcal{R} e \frac{N-\bar{N}}{\left(z-z_{j}\right)^{2}}
$$

giving representations (24) and (30). By the calculation of the solution of the exterior Neumann problem giving by (8), we have to find sums of the types

$$
\sum_{j=1}^{m} q_{j} \mathcal{R} e\left(z-z_{j}\right) \mathcal{R} e \frac{1}{z-z_{j}} \quad \text { and } \quad \sum_{j=1}^{m} q_{j} \mathcal{R} e\left(\log \left(z-z_{j}\right)\right) .
$$

In order to speed up the evaluation of the sums from (31) and (32), we would like to use the fast multipole method and, therefore, have to find for them the corresponding multipole and Taylor expansion, as well as appropriate translation and conversion operators. Such investigations for the terms

$$
\sum_{j=1}^{m} q_{j} \mathcal{R} e \frac{N_{j}}{z-z_{j}} \quad \text { and } \quad \sum_{j=1}^{m} q_{j} \mathcal{R} e\left(\log \left(z-z_{j}\right)\right)
$$

where $N_{j}$ is the complex representation of $\mathbf{n}(\mathbf{y})$, can be found, e.g., in [7], where they were presented in the context of the Laplace equation. Thus, we only will discuss in detail the terms

$$
\mathcal{R} e\left(N \sum_{j=1}^{m} \frac{q_{j}}{z-z_{j}}\right), \quad \sum_{j=1}^{m} q_{j} \mathcal{R} e\left(z-z_{j}\right) \mathcal{R} e \frac{1}{z-z_{j}}, \quad \text { and } \quad \sum_{j=1}^{m} q_{j} \mathcal{R} e\left(z-z_{j}\right) \mathcal{R} e \frac{N-\bar{N}}{\left(z-z_{j}\right)^{2}} .
$$

In order to obtain the multipole expansion for the terms in (33), we need

Lemma 4.1 Let $q_{0} \in \mathbb{R}$ and $N, z_{0} \in \mathbb{C}$ be given. Then for any $z$ with $|z|>\left|z_{0}\right|$ we have

(i) $\Phi_{z_{0}, 1}^{H}(z):=\mathcal{R} e\left(\frac{q_{0} N}{z-z_{0}}\right)=\mathcal{R} e\left(q_{0} N \sum_{k=0}^{\infty} \frac{z_{0}^{k}}{z^{k+1}}\right)$;

(ii) $\Phi_{z_{0}, 1}^{E}(z):=q_{0} \mathcal{R} e\left(z-z_{0}\right) \mathcal{R} e \frac{1}{z-z_{0}}=q_{0} \mathcal{R} e z \mathcal{R} e \sum_{k=0}^{\infty} \frac{z_{0}^{k}}{z^{k+1}}-q_{0} \mathcal{R} e \sum_{k=0}^{\infty} \frac{z_{0}^{k} \mathcal{R} e z_{0}}{z^{k+1}}$;

(iii) $\Phi_{z_{0}, 2}^{H}(z):=q_{0} \mathcal{R} e\left(z-z_{0}\right) \mathcal{R} e \frac{N-\bar{N}}{\left(z-z_{0}\right)^{2}}$

$$
=q_{0} \mathcal{R} e z \mathcal{R} e\left((N-\bar{N}) \sum_{k=0}^{\infty}(k+1) \frac{z_{0}^{k}}{z^{k+2}}\right)-q_{0} \mathcal{R} e\left((N-\bar{N}) \sum_{k=0}^{\infty}(k+1) \frac{z_{0}^{k} \operatorname{Re} z_{0}}{z^{k+2}}\right) ;
$$

(iv) $\Phi_{z_{0}, 2}^{E}(z):=\mathcal{R} e\left(q_{0} \log \left(z-z_{0}\right)\right)=\mathcal{R} e\left(q_{0}\left(\log z-\sum_{k=1}^{\infty} \frac{1}{k}\left(\frac{z_{0}}{z}\right)^{k}\right)\right)$.

Proof: With $|z|>\left|z_{0}\right|$ one obtain $(i)$ and (ii) by elementary calculations from the expansion

$$
\frac{1}{z-z_{0}}=\frac{1}{z}\left(1+\frac{z_{0}}{z}+\ldots+\left(\frac{z_{0}}{z}\right)^{n}+\ldots\right)=\sum_{k=0}^{\infty} \frac{z_{0}^{k}}{z^{k+1}}
$$

and (iii) from

$$
\frac{1}{\left(z-z_{0}\right)^{2}}=\frac{1}{z^{2}}\left(1+\frac{z_{0}}{z}+\ldots+\left(\frac{z_{0}}{z}\right)^{n}+\ldots\right)^{2}=\sum_{k=0}^{\infty}(k+1) \frac{z_{0}^{k}}{z^{k+2}} .
$$


In order to show the forth statement of the lemma, we note that $\log \left(z-z_{0}\right)-\log z=$ $\log \left(1-\frac{z_{0}}{z}\right)$ for $\left|\frac{z_{0}}{z}\right|<1$. Thus, (iv) follows immediately from the expansion

$$
\log \left(1-\frac{z_{0}}{z}\right)=(-1) \sum_{k=1}^{\infty} \frac{z_{0}^{k}}{k z^{k}}
$$

For the further investigations, we introduce the following notation:

$$
\begin{aligned}
& \Phi_{H_{1}}(z):=\sum_{j=1}^{m} \Phi_{z_{j}, 1}^{H}(z)=\mathcal{R} e\left(N \sum_{j=1}^{m} \frac{q_{j}}{z-z_{j}}\right) \\
& \Phi_{E_{1}}(z):=\sum_{j=1}^{m} \Phi_{z_{j}, 1}^{E}(z)=\sum_{j=1}^{m} q_{j} \mathcal{R} e\left(z-z_{j}\right) \mathcal{R} e \frac{1}{z-z_{j}}, \\
& \Phi_{H_{2}}(z):=\sum_{j=1}^{m} \Phi_{z_{j}, 2}^{H}(z)=\sum_{j=1}^{m} q_{j} \mathcal{R} e\left(z-z_{j}\right) \mathcal{R} e \frac{N-\bar{N}}{z-z_{j}}, \\
& \Phi_{E_{2}}(z):=\sum_{j=1}^{m} \Phi_{z_{j}, 2}^{E}(z)=\sum_{j=1}^{m} q_{j} \mathcal{R} e\left(\log \left(z-z_{j}\right)\right) .
\end{aligned}
$$

Theorem 4.2 (multipole expansion) Let $m \in \mathbb{N}, q_{j}, r \in \mathbb{R}$ and $N, z_{j} \in \mathbb{C}, j=1, \ldots, m$ be given. Then for any $z \in \mathbb{C}$ with $\left|z-z_{j}\right|>r$, the function $\Phi_{H_{1}}(z)$ is given by

$$
\Phi_{H_{1}}(z)=\mathcal{R} e\left(N \sum_{k=0}^{\infty} \frac{\tilde{a}_{k}}{z^{k+1}}\right) \quad \text { with } \quad \tilde{a}_{k}:=\sum_{j=1}^{m} q_{j} z_{j}^{k}
$$

It holds for any $p \in \mathbb{N}$

$$
\left|\sum_{k=p+1}^{\infty} \frac{\tilde{a}_{k}}{z^{k}}\right| \leq \frac{A}{|z|-r}\left|\frac{r}{z}\right|^{p+1} \text { with } A:=\sum_{j=1}^{m}\left|q_{j}\right| .
$$

Furthermore, for the function $\Phi_{H_{2}}(z)$, we get

$$
\Phi_{H_{2}}(z)=\mathcal{R} e z \mathcal{R} e\left((N-\bar{N}) \sum_{k=1}^{\infty} \frac{a_{k}}{z^{k+1}}\right)-\mathcal{R} e\left((N-\bar{N}) \sum_{k=1}^{\infty} \frac{a_{k}^{\prime}}{z^{k+1}}\right)
$$

with

$$
a_{k}:=k \sum_{j=1}^{m} q_{j} z_{j}^{k-1} \quad \text { and } \quad a_{k}^{\prime}:=k \sum_{j=1}^{m} q_{j} z_{j}^{k-1} \mathcal{R} e z_{j} .
$$

For any $p \in \mathbb{N}$, we obtain the estimates

$$
\left|\sum_{k=p+1}^{\infty} \frac{a_{k}}{z^{k+1}}\right| \leq \alpha_{1}\left|\frac{r}{z}\right|^{p} \quad \text { and }\left|\sum_{k=p+1}^{\infty} \frac{a_{k}^{\prime}}{z^{k+1}}\right| \leq \alpha_{2}\left|\frac{r}{z}\right|^{p}
$$

with

$$
\alpha_{i}:=\frac{A_{i}(r+(p+1)(|z|-r))}{|z|(|z|-r)^{2}}, i=1,2, \quad A_{1}:=\sum_{i=1}^{m}\left|q_{j}\right|, \quad A_{2}:=\sum_{i=1}^{m}\left|q_{j} \mathcal{R} e z_{j}\right| .
$$


Proof: One obtains the multipole expansion (38) from the definition of $\Phi_{H_{1}}(z)$ given in (34) and Lemma 4.1(i). In order to prove the estimate in (39), we substitute for $\tilde{a}_{k}$ the corresponding expression in (38) and obtain

$$
\left|\sum_{k=p+1}^{\infty} \frac{\tilde{a}_{k}}{z^{k}}\right| \leq A \sum_{k=p+1}^{\infty} \frac{r^{k}}{|z|^{k+1}} \leq \frac{A}{|z|}\left|\frac{r}{z}\right|^{p+1} \sum_{k=0}^{\infty}\left|\frac{r}{z}\right|^{k}=\frac{A}{|z|-r}\left|\frac{r}{z}\right|^{p+1} .
$$

For the multipole expansion of $\Phi_{H_{2}}(z)$, we find with Lemma 4.1(iii)

$$
\begin{aligned}
\Phi_{H_{1}}(z) & =\sum_{j=1}^{m} \mathcal{R} e(z) \mathcal{R} e\left((N-\bar{N}) \sum_{j=1}^{m} \frac{q_{j}}{\left(z-z_{j}\right)^{2}}\right)-\sum_{j=1}^{m} \mathcal{R} e\left(z_{j}\right) \mathcal{R} e \frac{q_{j}(N-\bar{N})}{\left(z-z_{j}\right)^{2}} \\
=: & T_{1}(z)-T_{2}(z),
\end{aligned}
$$

where

$$
\begin{aligned}
T_{1}(z) & =\mathcal{R} e z \mathcal{R} e\left((N-\bar{N}) \sum_{j=1}^{m} \frac{q_{j}}{z^{2}} \sum_{k=1}^{\infty} k \frac{z_{j}^{k-1}}{z^{k-1}}\right) \\
& =\mathcal{R} e z \mathcal{R} e\left((N-\bar{N}) \sum_{k=1}^{\infty} \frac{a_{k}}{z^{k+1}}\right) \quad \text { with } \quad a_{k}=k \sum_{j=1}^{m} q_{j} z_{j}^{k-1},
\end{aligned}
$$

and

$$
\begin{aligned}
T_{2}(z) & =\sum_{j=1}^{m} \mathcal{R} e\left((N-\bar{N}) \frac{q_{j} \mathcal{R} e z_{j}}{\left(z-z_{j}\right)^{2}}\right) \\
& =\operatorname{Re}\left((N-\bar{N}) \sum_{k=1}^{\infty} \frac{a_{k}^{\prime}}{z^{k+1}}\right) \quad \text { with } \quad a_{k}^{\prime}=k \sum_{j=1}^{m} q_{j} z_{j}^{k-1} \mathcal{R} e z_{j} .
\end{aligned}
$$

Further, we obtain the expressions in (42) and (43) by

$$
\left|\sum_{k=p+1}^{\infty} \frac{a_{k}}{z^{k+1}}\right| \leq \frac{A_{1}}{|z|^{2}}\left|\frac{r}{z}\right|^{p} \sum_{k=0}^{\infty}(k+p+1)\left|\frac{r}{z}\right|^{k}=\alpha_{1}\left|\frac{r}{z}\right|^{p}
$$

with

$$
\alpha_{1}=\frac{A_{1}(r+(p+1)(|z|-r))}{|z|(|z|-r)^{2}} .
$$

The following theorem presents the multipole expansion for the terms from (32).

Theorem 4.3 (multipole expansion) Let $m \in \mathbb{N}, q_{j}, r \in \mathbb{R}$ and $N, z_{j} \in \mathbb{C}, j=1, \ldots, m$ be given. Then for any $z \in \mathbb{C}$ with $\left|z-z_{j}\right|>r$, the function $\Phi_{E_{1}}(z)$ is given by

$$
\Phi_{E_{1}}(z)=\mathcal{R} e z \mathcal{R} e \sum_{k=0}^{\infty} \frac{a_{k}}{z^{k+1}} \quad-\quad \mathcal{R} e \sum_{k=0}^{\infty} \frac{a_{k}^{\prime}}{z^{k+1}}
$$


with

$$
a_{k}=\sum_{j=1}^{m} q_{j} z_{j}^{k} \quad \text { and } \quad a_{k}^{\prime}=\sum_{j=1}^{m} q_{j} z_{j}^{k} \mathcal{R} e z_{j}
$$

It holds for any $p \in \mathbb{N}$

$$
\left|\sum_{k=p+1}^{\infty} \frac{a_{k}}{z^{k}}\right| \leq \frac{A_{1}}{|z|-r}\left|\frac{r}{z}\right|^{p+1} \quad \text { with } \quad A_{1}:=\sum_{j=1}^{m}\left|q_{j}\right|
$$

and

$$
\left|\sum_{k=p+1}^{\infty} \frac{a_{k}^{\prime}}{z^{k}}\right| \leq \frac{A_{2}}{|z|-r}\left|\frac{r}{z}\right|^{p+1} \quad \text { with } \quad A_{2}:=\sum_{j=1}^{m}\left|q_{j} \mathcal{R} e z_{j}\right|
$$

Furthermore, for the function $\Phi_{E_{2}}(z)$, we get

$$
\Phi_{E_{2}}(z)=Q \log z+\sum_{k=1}^{\infty} \frac{\tilde{a}_{k}}{z^{k}} \quad \text { with } \quad Q=\sum_{j=1}^{m} q_{j}, \quad \tilde{a}_{k}=\sum_{j=1}^{m} \frac{-q_{j} z_{j}^{k}}{k} .
$$

For any $p \in \mathbb{N}$, we obtain the estimate

$$
\left|\Phi_{E_{2}}(z)-Q \log z-\sum_{k=1}^{p} \frac{\tilde{a}_{k}}{z^{k}}\right| \leq \frac{A}{1-|r / z|}\left|\frac{r}{z}\right|^{p+1} \quad \text { with } \quad A=\sum_{i=1}^{m}\left|q_{i}\right| .
$$

The corresponding proof proceeds on the lines of the proof of Theorem 4.2.

Statements, allowing us to manipulate the multipole expansion from Theorems 4.2 and 4.3 in a manner required by the fast multipole algorithm, are exactly the same as in [27] and [7]. Thus, we only present them here without giving the corresponding proofs. The following theorem provides a mechanism for shifting the center of a multipole expansion.

Theorem 4.4 Let $m \in \mathbb{N}, r, q_{j} \in \mathbb{R}, z_{0} \in \mathbb{C}$ be given and for any $z \in \mathbb{C}$ with $\left|z-z_{0}\right|<r$

$$
\begin{gathered}
\widetilde{\Phi}_{H_{1}, E_{1}}(z):=\sum_{k=0}^{\infty} \frac{a_{k}}{\left(z-z_{0}\right)^{k+1}} \quad \text { with } \quad a_{k} \quad \text { from } \quad \text { (45), } \\
\widetilde{\Phi}_{E_{2}}(z):=\tilde{a}_{0} \log \left(z-z_{0}\right)+\sum_{k=1}^{\infty} \frac{\tilde{a}_{k}}{\left(z-z_{0}\right)^{k}} \quad \text { with } \quad \tilde{a}_{k} \quad \text { from } \quad \text { (48), }
\end{gathered}
$$

and

$$
\widetilde{\Phi}_{H_{2}}(z):=\sum_{k=1}^{\infty} \frac{a_{k}^{\prime}}{\left(z-z_{0}\right)^{k+1}} \quad \text { with } \quad a_{k}^{\prime} \text { from }(41)
$$

Then for any $z \in \mathbb{C}$ with $|z|>R:=r+\left|z_{0}\right|$,

$$
\begin{gathered}
\widetilde{\Phi}_{H_{1}, E_{1}}(z)=\sum_{l=1}^{\infty} \frac{b_{l}}{z^{l}} \quad \text { with } \quad b_{l}=\sum_{k=1}^{l} a_{k} z_{0}^{l-k}\left(\begin{array}{l}
l-1 \\
k-1
\end{array}\right), \\
\widetilde{\Phi}_{E_{2}}(z)=\tilde{a}_{0} \log z+\sum_{l=1}^{\infty} \frac{\tilde{b}_{l}}{z^{l}} \quad \text { with } \quad \tilde{b}_{l}=\sum_{k=1}^{l} \tilde{a}_{k} z_{0}^{l-k}\left(\begin{array}{l}
l-1 \\
k-1
\end{array}\right)-\frac{\tilde{a}_{0} z_{0}^{l}}{l},
\end{gathered}
$$


and

$$
\widetilde{\Phi}_{H_{2}}(z)=\sum_{l=1}^{\infty} \frac{b_{l}^{\prime}}{z^{l+1}} \quad \text { with } \quad b_{l}^{\prime}=\sum_{k=1}^{l} a_{k}^{\prime} z_{0}^{l-k}\left(\begin{array}{l}
l \\
k
\end{array}\right),
$$

respectively, and for any $p \in \mathbb{N}$,

$$
\begin{gathered}
\left|\widetilde{\Phi}_{H_{1}, E_{1}}(z)-\sum_{l=1}^{p} \frac{b_{l}}{z^{l}}\right| \leq\left(A /\left(1-\left|\frac{R}{z}\right|\right)\right)\left|\frac{R}{z}\right|^{p+1}, \\
\left|\widetilde{\Phi}_{E_{2}}(z)-\tilde{a}_{0} \log z-\sum_{l=1}^{p} \frac{\tilde{b}_{l}}{z^{l}}\right| \leq\left(A /\left(1-\left|\frac{R}{z}\right|\right)\right)\left|\frac{R}{z}\right|^{p+1}
\end{gathered}
$$

with $A=\sum_{i=1}^{m}\left|q_{j}\right|$, and

$$
\left|\widetilde{\Phi}_{H_{2}}(z)-\sum_{l=1}^{p} \frac{b_{l}^{\prime}}{z^{l+1}}\right| \leq \alpha\left|\frac{R}{z}\right|^{p+1}
$$

with

$$
\alpha=\frac{A(R+(p+1)(|z|-R))}{|z|(|z|-R)^{2}}, i=1,2, \quad A=\sum_{i=1}^{m}\left|q_{j} \mathcal{R} e z_{j}\right|
$$

The next theorem describes how to convert the shifted expansion into a local (Taylor) expansion in a circular region of analyticity.

Theorem 4.5 Let $m \in \mathbb{N}, c, r, q_{j} \in \mathbb{R}$ and $z_{0} \in \mathbb{C}, j=1, \ldots m$, be given with $\left|z_{0}\right|>(c+1) r$ and $\left|z-z_{0}\right|<r$ for all $z \in \mathbb{C}$. Then, the multipole expansions from (50)-(52) converge in the interior of the circle of radius $r$ with center at the origin and can be represented by

$$
\begin{gathered}
\widetilde{\Phi}_{H_{1}, E_{1}}(z)=\sum_{l=0}^{\infty} b_{l} z^{l} \quad \text { with } \quad b_{l}=\frac{1}{z_{0}^{l}} \sum_{k=1}^{\infty}\left(\begin{array}{c}
l+k-1 \\
k-1
\end{array}\right) \frac{a_{k}}{\left(-z_{0}\right)^{k}}, \quad a_{k} \quad \text { from } \\
\widetilde{\Phi}_{E_{2}}(z)=\sum_{l=0}^{\infty} \tilde{b}_{l} z^{l} \quad \text { with } \quad \tilde{b}_{0}=\sum_{k=1}^{\infty} \frac{\tilde{a}_{k}}{\left(-z_{0}\right)^{k}}+\tilde{a}_{0} \log \left(-z_{0}\right), \\
\tilde{b}_{l}=\frac{1}{z_{0}^{l}} \sum_{k=1}^{\infty}\left(\begin{array}{c}
l+k-1 \\
k-1
\end{array}\right) \frac{\tilde{a}_{k}}{\left(-z_{0}\right)^{k}}-\frac{\tilde{a}_{0}}{l z_{0}^{l}} \quad \text { for } \quad l \geq 1, \quad \tilde{a}_{k} \text { from }(48),
\end{gathered}
$$

and

$$
\widetilde{\Phi}_{H_{2}}(z)=\sum_{l=0}^{\infty} b_{l}^{\prime} z^{l} \quad \text { with } \quad b_{l}^{\prime}=\frac{1}{z_{0}^{l+1}} \sum_{k=1}^{\infty}(-1)\left(\begin{array}{c}
l+k \\
k
\end{array}\right) \frac{a_{k}^{\prime}}{\left(-z_{0}\right)^{k}}, \quad a_{k}^{\prime} \quad \text { from }
$$

Furthermore, for any $p \in \mathbb{N}, p \geq \max (2,2 c /(c-1))$, and Euler's constant $e$, error bounds for the truncated series are given by

$$
\left|\widetilde{\Phi}_{H_{1}, E_{1}}(z)-\sum_{l=0}^{p} b_{l} z^{l}\right| \leq \frac{4 A e p(c+1)}{r c(c-1)}\left(\frac{1}{c}\right)^{p+1} \quad \text { with } \quad A=\sum_{i=1}^{m}\left|q_{j}\right|
$$




$$
\left|\widetilde{\Phi}_{E_{2}}(z)-\sum_{l=0}^{p} \tilde{b}_{l} z^{l}\right| \leq \frac{A\left(4 e p(c+1)+c^{2}\right)}{c(c-1)}\left(\frac{1}{c}\right)^{p+1} \quad \text { with } \quad A=\sum_{i=1}^{m}\left|q_{j}\right|
$$

and

$$
\left|\widetilde{\Phi}_{H_{2}}(z)-\sum_{l=0}^{p} b_{l}^{\prime} z^{l}\right| \leq \frac{4 A e p^{2}(c+1)}{r^{2}(p+c-1)^{2}(c-1)}\left(\frac{1}{c}\right)^{p+1} \quad \text { with } \quad A=\sum_{i=1}^{m}\left|q_{j} \mathcal{R} e z_{j}\right| .
$$

Proof: One finds the proof of (61) and (64), e.g., in [2]. The proof of the other statements proceeds analog and was shown, e.g., in [27].

The following lemma describes a translation operation for the local (Taylor) expansion. The operation is exact with a finite number of terms. Therefore, no error bound is required.

Lemma 4.6 For any $z, z_{0}, a_{k} \in \mathbb{C}(k=0,1, \ldots, n)$, we have

$$
\sum_{k=0}^{n} a_{k}\left(z-z_{0}\right)^{k}=\sum_{l=0}^{n}\left(\sum_{k=l}^{n} a_{k}\left(\begin{array}{l}
k \\
l
\end{array}\right)\left(-z_{0}\right)^{k-l}\right) z^{l} .
$$

Remark: The generalization of the presented results to the Stokes equations in three dimensions is the object of the forthcoming work. In such a case, the multipole expansion can be obtained in the terms of spherical harmonics (cf. [8]).

\section{$5 \quad$ Numerical results}

For testing purposes, we assume a domain $G$ with the boundary defined by

$$
\begin{aligned}
& x(t):=\cos (2 \pi t), \\
& y(t):=\left\{\begin{array}{c}
\sin (2 \pi t), \quad t \in\left[0, \frac{1}{8}\right] \cup\left[\frac{3}{8}, 1\right], \\
-\frac{\sqrt{2}}{4}\left(\cos ^{4}(2 \pi t)+\cos ^{2}(2 \pi t)-\frac{11}{4}\right), \quad t \in\left(\frac{1}{8}, \frac{3}{8}\right),
\end{array}\right.
\end{aligned}
$$

$t \in[0,1]$, which is discretized by $m$ discretization points $\left(x\left(t_{i}\right), y\left(t_{i}\right)\right)$ with $t_{i}:=i / m, i=$ $1, \ldots, m$. We set the density function $\mathbf{q}=\mathbf{1}$ in all the points and computed the velocity part of the hydrodynamical single layer potential (9) and its normal stress operator (12).

At first, we are interested in the connection between the number $p$ of terms in the expansions and the maximal relative error in FMM approximation of a hydrodynamical single layer potential $E \mathbf{q}$ and its normal stress $H \mathbf{q}$ obtained at any of the discretization points. This error is defined via the formulas

$$
\varepsilon_{r e l}^{E}:=\max _{i=1, \ldots, n}\left|\frac{\Phi_{d i r}^{E}\left(z_{i}\right)-\Phi_{F M M}^{E}\left(z_{i}\right)}{\Phi_{d i r}^{E}\left(z_{i}\right)}\right| \quad \text { and } \quad \varepsilon_{r e l}^{H}:=\max _{i=1, \ldots, n}\left|\frac{\Phi_{d i r}^{H}\left(z_{i}\right)-\Phi_{F M M}^{H}\left(z_{i}\right)}{\Phi_{d i r}^{H}\left(z_{i}\right)}\right| \text {, }
$$

where $\Phi_{d i r}^{E}\left(z_{i}\right)$ and $\Phi_{d i r}^{H}\left(z_{i}\right)$ denote a hydrodynamical single layer potential and its normal stress obtained by the direct calculation (in double precision), $\Phi_{F M M}^{E}\left(z_{i}\right)$ and $\Phi_{F M M}^{H}\left(z_{i}\right)$ - a hydrodynamical single layer potential and its normal stress computed using the fast multipole method. The corresponding results for $m=1000$ are presented in Table 1 . 
Table 1: Maximal relative error in the FMM approximation of $E \mathbf{q}$ and $H \mathbf{q}$

\begin{tabular}{|c|c|c|}
\hline$p$ & $\varepsilon_{\text {rel }}^{E}$ & $\varepsilon_{\text {rel }}^{H}$ \\
\hline \hline & $1.23 \cdot 10^{-3}$ & $7.26 \cdot 10^{-3}$ \\
5 & $6.12 \cdot 10^{-6}$ & $8.49 \cdot 10^{-5}$ \\
10 & $1.48 \cdot 10^{-7}$ & $3.32 \cdot 10^{-6}$ \\
15 & $4.28 \cdot 10^{-8}$ & $1.13 \cdot 10^{-6}$ \\
16 & $2.51 \cdot 10^{-8}$ & $6.85 \cdot 10^{-7}$ \\
17 & $1.15 \cdot 10^{-9}$ & $4.32 \cdot 10^{-8}$ \\
20 & & \\
& \\
\hline
\end{tabular}

We compare now the speed and accuracy of the calculation using fast multipole method to those of the direct method. We perform the numerical calculation in two ways in double precision arithmetic: directly, e.g., via the direct matrix-vector multiplication, and via the fast multipole method of Greengard and Rokhlin. The number of terms in the expansions $p$ was set to 17 in order to guarantee roughly 6 -digit accuracy of the results. The results for the CPU time (in seconds) and the storage (in MB) costs corresponding to the calculation of $E \mathbf{q}$ are summarized in Table 2, and corresponding to the calculation of $H \mathbf{q}$ are in Table 3. The first column of the tables contains the number $m$ of discretization points, in the second column, $l$ denotes the number of refinements, the third and forth columns present the CPU time in seconds for the direct matrix-vector multiplication $t_{d i r}$ and for the computation of matrix-vector product via FMM $t_{F M M}$, respectively. In the last column, we present the information about the additional storage that was needed for FMM in compare to the direct method.

Table 2: Timing and storage results for the calculation of $E \mathbf{q}$

\begin{tabular}{|c|c|c|c|c|}
\hline$m$ & $l$ & $t_{d i r}^{E}$ & $t_{F M M}^{E}$ & $\begin{array}{c}\text { additional } \\
\text { storage }\end{array}$ \\
\hline \hline & & & & \\
4000 & 5 & 5.01 & 0.84 & 0.28 \\
8000 & 6 & 20.02 & 1.82 & 0.04 \\
16000 & 7 & 88.19 & 3.96 & -2.00 \\
32000 & 8 & 400.17 & 8.40 & -12.24 \\
64000 & 9 & 1984.21 & 17.98 & -57.60 \\
& & & & \\
\hline
\end{tabular}

Table 3: Timing and storage results for the calculation of $H \mathbf{q}$

\begin{tabular}{|c|c|c|c|c|}
\hline$m$ & $l$ & $t_{\text {dir }}^{H}$ & $t_{F M M}^{H}$ & $\begin{array}{c}\text { additional } \\
\text { storage }\end{array}$ \\
\hline \hline & & & & \\
4000 & 5 & 4.05 & 1.90 & 0.31 \\
8000 & 6 & 17.56 & 4.09 & 0.15 \\
16000 & 7 & 83.19 & 8.24 & -1.41 \\
32000 & 8 & 381.21 & 17.77 & -10.00 \\
64000 & 9 & 1784.23 & 38.08 & -49.33 \\
\hline
\end{tabular}

The numerical experiments show that for the cases considered, the fast multipole method was more efficient then direct calculation. From Tables 2 and 3, one can made the observation that in the case of the fast multipole method, the CPU time grows almost linearly with the number of discretization points. Moreover, for a number of discretization points more above 16000, the savings in memory are also significant. 


\section{References}

[1] Borchers, W.; Varnhorn, W.: On the boundedness of the Stokes semigroup in twodimensional exterior domains. Math. Z. 213, 275-300 (1993)

[2] Carrier, J.; Greengard L.; Rokhlin, V.: A fast algorithm for particle simulations. J. Sci. Stat. Comp. 9, 669-686 (1988)

[3] Ethridge, F.; Greengard, L.: A new fast multipole accelerated Poisson solver in two dimensions. SIAM J. Sci. Comput. 23, 3, 741-760 (2001)

[4] Gáspár, C.: A multipole expansion technique in solving boundary integral equations. Comput. Methods Appl. Mach. Eng. 157, 289-297 (1998)

[5] Greengard, L.; Rokhlin, V.: Rapid evaluation of potential fields in three dimensions. In: Vortex Methods, edited by C. Anderson and C. Greengard, Lecture Notes in Mathematics. Berlin: Springer (1988)

[6] Greengard, L.; Kropinski, M.C.; Mayo, A.: Integral equation methods for Stokes flow and isotropic elasticity in the plane. J. Comp. Phys. 125, 403-414 (1996)

[7] Greengard, L.; Rokhlin, V.: A fast algorithm for particle simulations. J. Comp. Phys. 135, 280-292 (1997a)

[8] Greengard, L.; Rokhlin, V.: A new version of the fast multipole method for the Laplace equation in three dimensions. Acta Numerica 6, 229 (1997b)

[9] Greengard, L.; Kropinski, M.C.: An integral equation approach to the incompressible Navier-Stokes equations in two dimensions. SIAM J. Sci. Comput. 20, 318-336 (1998)

[10] Hsiao, G.C.: Integral representation of solutions for two-dimensional viscous flow problems. Integral Equations and Operator Theory 5, 533-547 (1982)

[11] Hsiao, G.C.; Kress, R.: On an integral equation for the two-dimensional exterior Stokes problem. Appl. Num. Math. 1, 77-93 (1985)

[12] Jasnow, M.A.; Symm, G. T.:Integral Equation Methods in Potential Theory and Elastostatics. Academic Press, New York (1977)

[13] Khoromskij, B.N.; and Wittum, G.: Numerical Solution of Elliptic Differential Equations by Reduction to the Interface. Research monograph, LNCSE, No. 36, Springer-Verlag (2004).

[14] Ladyzhenskaya, O.A.: The mathematical theory of viscous incompressible flow. New York: Gordon and Breach Science Publishers Inc. (1969)

[15] Mayo, A.; Greebaum, A.: Fast parallel iterative solution of Poisson's and the biharmonic equations on irregular regions. SIAM J. Sci. Statist. Comput. 13, 101-118 (1992)

[16] Mikhlin, S.G.: Integral Equations. Pergamon, London (1957)

[17] Odquist, F.K.G.: Über die Randwertaufgaben in der Hydrodynamik zäher Flüssigkeiten. Math. Z. 32, 329-375 (1930)

[18] Of, G.: A fast multipole boundary element method for the symmetric boundary integral formulation in linear elastostatics. Comp. Fluid Solid Mech., 540-543 (2003) 
[19] Rahola, J.: Diagonal Forms of the Translation operators in the Fast Multipole Algorithm for Scattering Problems. BIT 36, 333-358 (1996)

[20] Pérez-Jordá, J.M.: Yang, W.: A simple $O(N \log N)$ algorithm for the rapid evaluation of particle-particle interactions. Chem. Phys. Letters 247, 484-490 (1995)

[21] Popov, A.N.: Application of Potential Theory to the Solution of a Linearized System of Navier-Stokes Equations in the Two-Dimensional Case. In: "Proceedings of the Steklov Institute of Mathematics 116 (Ed.: O.A. Ladyzhenskaja)", Providence R.I.: Amer. Math. Soc. 167-186 (1973)

[22] Pozrikidis, C.: Boundary Integral and Singularity Methods for Linearized Viscous Flow. Cambridge Univ. Press, Cambridge (1992)

[23] Rokhlin, V.: Rapid solution of integral equations of classical potential theory. J. Comp. Phys. 60, 187 (1985)

[24] Rokhlin, V.: Rapid solution of integral equations of scattering theory in two dimensions. J. Comp. Phys. 86, 414 (1990)

[25] Samrowski, T.S.: A multipole method for the Rapid Solution of the Stationary Linearized Stokes System. PAMM 5, 839-840 (2005)

[26] Samrowski, T.S.: Eine Multipole Methode für die Gleichungen von Stokes. Dissertation Universität Kassel (2005)

[27] Samrowski, T.S.: Justification of the Fast Multipole Method for the Stokes System. submitted J. Math. Sci. (N.Y.) (2011)

[28] Song, J.M.; Chew, W.C.: Multilevel fast multipole algorithm for electromagnetic scattering by large complex objects. IEEE Trans. Antennas Propag. 45(10) 1488-1496 (1997)

[29] Varnhorn, W.: The Stokes Equations. Berlin: Akademie (1994)

[30] Zhenhan Yao, Haitao Wang, Pengbo Wang, Ting Lei: Some Applications of Fast Multipole Boundary Element Method, Department of Engineering Mechanics. Tsinghua University, Beijing (2004) 